\title{
Effect of fabric electron radiation on increasing the antibacterial coating and perfume longevity
}

\author{
M. Askarbioki ${ }^{1,2}$, M. B. Zarandi ${ }^{1}$, S. Khakshournia, ${ }^{2,}$, S. P. Shirmardi ${ }^{2}$, S. Kargar ${ }^{3}$, A. Amooee ${ }^{3}$, \\ M. Sharifian ${ }^{1}$, S. Ghafoorzadeh ${ }^{2}$ \\ ${ }^{I}$ Atomic \& Molecular Group, Physics Factually, Yazd University, Yazd, Iran \\ ${ }^{2}$ Nuclear Science and Technology Research Institute (NSTRI), Tehran, Iran \\ ${ }^{3}$ Department of General Surgery, Shahid Sdoughi Hospital, Shahid Sdoughi University of Medical Sciences, Yazd, Iran
}

\begin{abstract}
In this paper, we studied the effect of electron beam irradiation on the fabric to increase longevity of antibacterial coating and perfume release and measure it using the optical properties of the fabric. In other words, instead of using chemical compounds in the antibacterial coating and perfume structure, the change in the structural properties of the fabric as a substrate of antibacterial coating and perfume was examined. Three different types of fabrics, including fabric with polyester and cotton, fabric with felt and flannelette, and fabric with flannelette and cotton floss were irradiated at different doses without alcohol and in the presence of alcohol (96\% ethanol) at an energy of $10 \mathrm{MeV}$ with an electron beam of the Rhodotron accelerator TT200. Then, these three types of fabrics were impregnated with antibacterial coating and perfume after washing with cold water. Finally, the longevity of antibacterial coating and perfume on them was measured by using the Particle Density Reflection Parameters and He-Ne laser with a wavelength of $632 \mathrm{~nm}$ and a power of $5 \mathrm{~mW}$. Experimental results showed that electron beam irradiation of the fabric in the presence of alcohol enhanced this property. Keywords: Antibacterial coating and antibacterial coating and perfume longevity; Electron beam irradiation; He-Ne laser; Aromatic fabric absorption coefficient.
\end{abstract}

\section{INTRODUCTION}

Today, researchers have a vast desire to increase the antibacterial coating and perfume's longevity, and there are different methods to develop it [1] A wide range of studies have been carried out on transmitting aromatic scents. In most of them, the method of work the synthesis of materials, and the synthesis of materials and measuring them are dealt with. Earlier in the past two decades, a group of researchers has reported increasing the time of antibacterial coating and perfume release using chemical processes and structures [2]. In this research, the successful use of tree-like networks has resulted in greater longevity of antibacterial coating and perfume. The high-performance liquid chromatography (HPLC) has measured a small amount of antibacterial coating, and perfume extraction has been measured by the highperformance liquid chromatography (HPLC). Afterwards, the effect of UV radiation on the diffusion of various components of the antibacterial coating and perfume structure was studied using a Nuclear Magnetic Resonance 
(NMR) device [3]. Then the use of micro-capsulation chemical techniques was reported [4] in which researchers have shown the importance of the method of preparing these microcapsules containing aromatic particles and determined the time of antibacterial coating and perfume emission by measuring the dimensions of these microcapsules by the Scanning Electron Microscopy (SEM) at specific time intervals. In addition, the use of delivery systems in aqueousaromatic solutions and their control by organic molecules for improving the antibacterial coating and perfume's longevity have been reported [5]. With increasing the use of the microcapsule synthesis technique, the researchers focused on the better construction and more accurate measurement, so that the synthesis of biodegradable microcapsules by non-toxic compounds and their precise characterization were reported [6]. In this study to accurately measure the size of the microcapsules and rate of its reduction due to the release of antibacterial coating and perfume, the UV-Visible Spectroscopy, Fourier Transform Infrared (FTIR) Spectroscopy, and Environmental Scanning Electron Microscope (ESEM) were used. In another study, focusing on the accurate measurement of the emission rate, the use of two methods of thermal weighing and electric nose has been reported [7]. New measurements for the diffusion of antibacterial coating and perfume with different instruments continued until the use of SEM with Laser Scanning Co-focal was proposed [8]. In this work, in addition to the innovation in measuring instruments, the production of microcircuits has also been reported to improve the performance of antibacterial coating and perfume release.
Recently, some researchers have used a comprehensive measurement tool, including the $\mathrm{x}$-ray diffraction method, thermal imaging and FTIR to examine the lattice structure of their product, and finally using the Static Headspace Gas Chromatography, they have analyzed the rate of antibacterial coating and perfume emission [9]. In this way, several works based on using organicmetal materials and assessment by the GC/Mass [10]. natural fragrances with essential oils, dry spray and FTIR assessment [11,12], and vacuum drying techniques with Spry Drying [13] were reported. Then a new method was proposed in which the moisture-activated odour release from fragrance inclusion complexes was visualized by using a fluorescence imaging system. The visualization was based on the fluorescence change of encapsulated fragrances induced by the odour release [14] Kuhnt et al. [15] studied the fragrance release in an aqueous solution by using ${ }^{1} \mathrm{H}-\mathrm{NMR}$ spectroscopy as a function of $\mathrm{pH}$. With the expansion and willingness to use nanotechnology, research on the antibacterial coating and perfume longevity rate based on the use of nanoparticles increased. A successful report was released on the use of nanoparticles to enhance the longevity of fragrance without having any negative effect on real and natural environments [17]. In this research, nanocomposites containing aromatic particles were synthesized and then applied to the human microbial media. The transmission electron microscope (TEM) images and dynamic light scattering results showed the success of this technique [18].

In recent years, these research topics have been applied in practice [19], and the production of light-sensitive microcapsules [20] and the 
hydrogel-cell structure of a microfluid in a microcapsule have been successfully reported [21]. The underlying aim of nearly all recent studies is to optimally use the aromatic compounds with a minimal cost.

Antibacterial coating and perfume are usually released from human skin; hence, a majority of research studies have been conducted on their chemical structures to increase their durability. Recently, however, many companies and brands tend to have the smell of antibacterial coating and perfume on their logo or brand for a long time, when it is first stuck on their clothes. Therefore, these companies are looking for yarns and fabrics, causing higher durability of antibacterial coating and perfume and antibacterial materials, regardless of their type [22,23]. The use of nanotechnology, as mentioned above, specific polymers, and various processes is an example of their attempt to increase the antibacterial coating and perfume durability [22,24]. Also, performing this experiment and presenting the findings of this study can greatly contribute to the spread of durable, low-cost and controlled materials from polymer and fabric substrates. These include the release of fragrance and antibacterial molecules in restrooms, hospital care and treatment rooms [24].

The modification of the structure and surface of a variety of fabrics by ionizing radiation has been investigated. Some researchers have reported the use of gamma radiation to prepare the fabric surface for the formation of suitable secondary bonds [25]. In this study, acrylic acid was grafted onto a polypropylene fabric, and then the surface of the fabric was modified using the ferrous ion and sulfuric acid compound, and the fabric was prepared to form new bonds. Similarly, the gamma radiation of cotton fabric surface in the presence of temperature-sensitive poly- $\mathrm{N}$-isopropylacrylamide has been studied [26]. The use of gamma radiation to form suitable bonds between fabric fibers and a variety of specific polymers to optimize antimicrobial properties has also been reported frequently [26,27].

The use of ionizing e-beam radiation has also been examined to form suitable functional groups for the absorption of metal ions [28]. In this research, a non-woven polypropylene fabric coated with polyethylene was exposed to $\mathrm{N}_{2}$ gas under an electron beam of $200 \mathrm{kGy}$ to be prepared to absorb the metal ions needed to optimize the surface properties.

A thorough investigation has been carried out on the study of gamma radiation on the chemical and physical structure of cotton [29-31]. Comprehensive reports have been published on the benefits of UV light for improving the wrinkle resistance and the flame resistance properties in cotton [32]. In another study, the differences between gamma radiation, electron beam, and neutron radiation on cotton were compared and investigated [33]. Cotton fibers behave differently in swelling when exposed to these beams; gamma and electron radiation cause fibrillation in cotton during beating in water.

Radiation of the ${ }^{60} \mathrm{Co}$ causes the moduli of elasticity of the polyester filaments to increase, while the solubility of the filaments decreases and the melting point remains the same [34]. Electron irradiation causes changes in the tensile strength of polyester polymers such that for doses below 100 $\mathrm{kGy}$, its amount is less than 15\% [35]. Long-term $\mathrm{UV}$ radiation reduces the bending strength of 
polyester fabrics and accelerates the corrosion process of polymer [36].

In this paper, a new method for increasing the longevity of antibacterial coating and perfume from cotton and polyester fabrics as well as measuring this quantity is presented. The method used in this study is the application of electron irradiation on the fabric samples put in a vessel containing $96 \%$ ethanol. Although so far, the focus has been on changing and optimizing the antibacterial coating and perfume's structure, this work attempts to demonstrate the importance of the fabric as the antibacterial coating and perfume release bed.

\section{MATERIALS AND METHODS}

Adding moisture to the tissue or particles forming a material to reduce the refractive index changes across the common boundary of the material with air makes that material appear transparent [37]. Thus the fragrance molecules which have a refractive index close to water, by being added to the fabric will make it transparent. The changes caused by the release of fragrance molecules over time will reduce transparency, naturally increasing the absorption, and hence the variation in the intensity of laser light passing through the aromatic fabric. Therefore, by measuring the changes in the absorption coefficient of aromatic fabrics, one can obtain the rate of release of antibacterial coating and perfume with time. Since the structure of the textile fiber does not change due to the aroma composition, hence the change of its absorption coefficient over time indicates the presence or absence of fragrance molecules.

Adding antibacterial coating and perfume causes no change in the physical and chemical structure of the fabric molecules, and its refractive index and absorption coefficient does not change. However, as mentioned in previous studies, when structural fibers of fabric molecules are formed in millimeters and micrometers and are exposed to moisture, they appear to be brighter [37].

In Fig. 1, two similar samples of a type of fabric are shown. One sample was wetted with alcohol (Fig. 1-a), and the other sample was completely dry (Fig. 1-b). Two samples were located at a distance of $2 \mathrm{~m}$ from a white LED with a power of $3 \mathrm{~W}$.

As shown in Fig. 1, the wet fabric (Fig. 1-a) looks brighter. In other words, the wet cloth moves away from its diffraction-like state and transmits more light. Indeed, the alcohol in the antibacterial coating and perfume has a refractive index which is closer to that of the water because its absorption for visible waves, especially the $\mathrm{He}-\mathrm{Ne}$ laser wavelength at $632 \mathrm{~nm}$, is much less than for fragrance molecules.
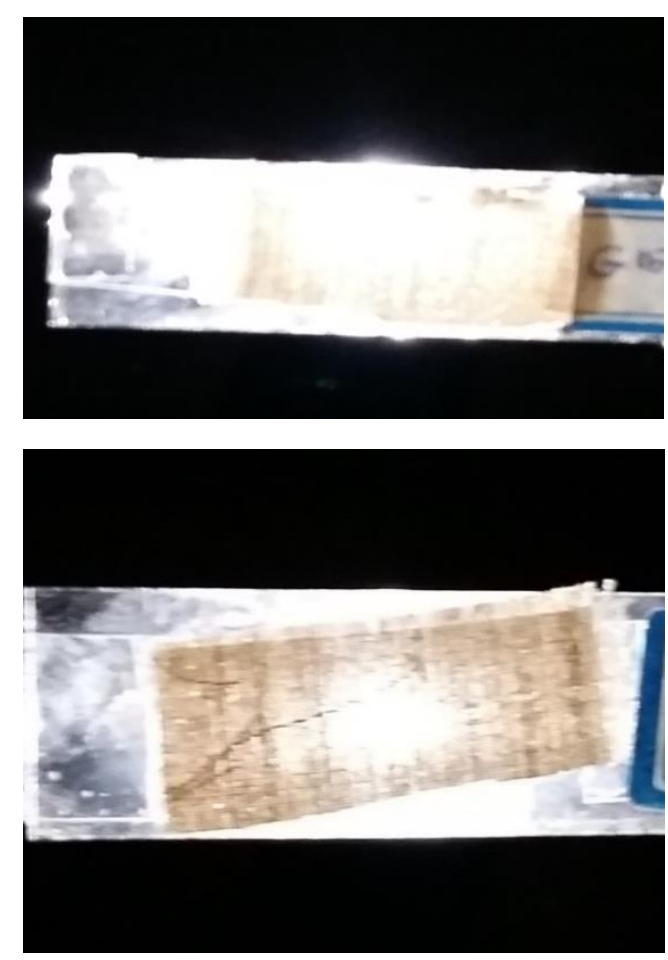

Fig. 1. Two samples of fabrics: a) Wet fabric; b) Dry fabric. 
What's important is the antibacterial coating and perfume's release time, not its solvent. When the antibacterial coating and perfume is sprayed, its evaporation rate is different depending on its solvent, which is predominantly of an alcoholic structure. In this study, the evaporation rate of fragrance molecules was examined. To this end, the antibacterial coating and perfume (Beaumont White Gold Amordad for men, Fragrantica Inc, San Diego, CA United States) release rates were followed using a conventional FTIR spectrometer on a conventional $\mathrm{KBr}$ crystal bed. The FTIR absorption spectrum of the antibacterial coating and perfume used in this study is presented in Figure 2.

In the FTIR spectrum, the absorption is investigated, and it is calculated from the equation: Absorption $=1-($ Transmission + Reflection $)$. A drop of antibacterial coating and perfume is placed on a $\mathrm{KBr}$ pellet and the way that the antibacterial coating and perfume is released in the air between the time duration of $\mathrm{t}=0$ and $25 \mathrm{~min}$ is shown in Figure 2.

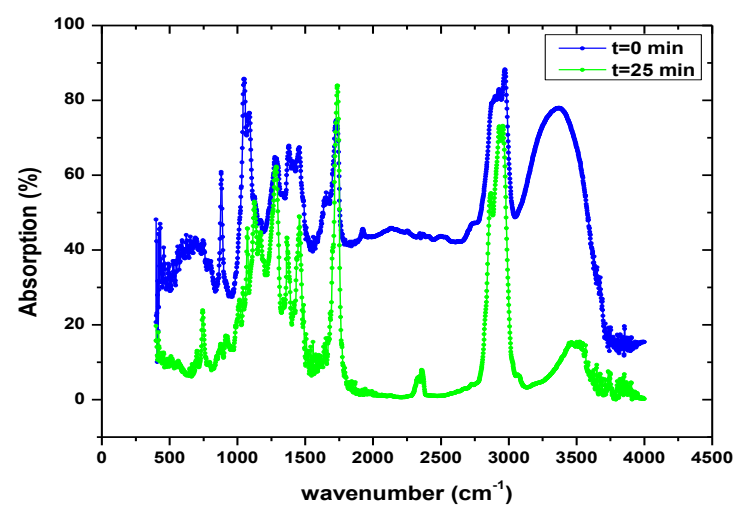

Fig. 2. FTIR absorption spectrum diagram of the antibacterial coating and perfume used in the experiment.
Blue dashed line (-॰-) actually represents the chemical structure of the antibacterial coating and perfume used in this study at spray time of $t=0$. Green dashed line (-॰-) also shows the chemical structure of the remaining molecules at $\mathrm{t}=25 \mathrm{~min}$. In Fig. 2, the spectrum of alcoholic particles with a center line of $3500 \mathrm{~cm}^{-1}$ has dropped by $62 \%$ over time; however, the spectrum of fragrance molecules remained unchanged on other lines. With regard to spectral variations of alcoholic particles on the central line $3500 \mathrm{~cm}^{-1}$ over time, it can be inferred that the solvent alcohol evaporation rate is greater than the evaporation rate of the fragrance molecules. What is measured using an optical method is the release rate of all antibacterial coating and perfume particles over time, the higher rate of which is for the fragrance molecules.

The diagram represented in Fig 2 shows the spectrum of the antibacterial coating and perfume used in this research. After 25 minutes, a high amount of the alcohol with the central line of 3500 $\mathrm{cm}^{-1}$ evaporates, and its amount decreases, whereas the other fragrance molecules, which are represented by the remaining lines in the diagram, do not change such rapidly. As shown in Fig. 2, the indicator peaks include the alcoholic $\mathrm{OH}$ on 3500 $\mathrm{cm}^{-1}$, and on the left side, the $\mathrm{CH}$ alkane group on $3000 \mathrm{~cm}^{-1}$, Carbonyl on $1700 \mathrm{~cm}^{-1}$, and C-O on $1100 \mathrm{~cm}^{-1}$. This evidence suggests that the antibacterial coating and perfume used in this study are aliphatic sterile. After 25 minutes, the indicator peak of the $\mathrm{OH}$ group decreased, showing the evaporation of the weak alcoholic group. The emergence of the indicator peak on $700 \mathrm{~cm}^{-1}$ indicates the presence of alkaline $\mathrm{CH} 2$ in the antibacterial coating and perfume. According to 
this, the laboratory arrangement was made as shown in Fig. 3.

In this optical setup, a He-Ne laser with a wavelength of $632 \mathrm{~nm}$ and a $5 \mathrm{~mW}$ power and two optical detectors TCS3200-E11 were used. Although the He-Ne gas laser has relatively stable power, slight changes in its power can cause systematic and random measurement errors. The use of He-Ne laser is not for checking the release of a specific molecule of the antibacterial coating and perfume from the fabric, but it is aimed at using coherent light of laser to indicate the degree of antibacterial coating and perfume release and drying rate of the wet fabric. In other words, instead of using expensive methods to determine the degree of antibacterial coating and perfume release and drying rate of the wet fabric, the light diffraction phenomenon was used [37].

Therefore, we used a BS (50:50 beam splitter) to read the initial power of the laser beam $\left(I_{0}=2 I_{1}\right)$ and transmitting power $\left(I_{2}\right)$. All samples were placed in the microscope slides after being washed with the cold water at $15^{\circ} \mathrm{C}$ and immersed in an antibacterial coating and perfume with enough spraying it on the samples. These specimens were kept separately at $23 \mathrm{C}$ temperature and tested for 30 minutes after immersion. The results of the variation of intensities of $I_{1}$ and $I_{2}$ were recorded at these intervals and finally, changes in the absorption coefficient of various samples, including antibacterial coating and perfumeimpregnated fabrics for the He-Ne laser $632 \mathrm{~nm}$ were measured over time. In this study, the thickness of all the fabrics was the same, $0.2 \mathrm{~mm}$, and the absorption coefficient to be measured was given by

$$
\alpha\left(m^{-1}\right)=\frac{-1}{0.0002} \ln \left(\frac{I_{2}}{I_{0}}\right)
$$

The absorption coefficient was measured at three points in their cross-sections using the $\mathrm{He}-\mathrm{Ne}$ laser with the following spatial profile (Fig. 4) for the fabric samples $S_{1}, S_{2}$ and $S_{3}$ in the presence/absence of alcohol. The measurement was made within the $1.34 \mathrm{~mm}$ region corresponding to the FWHM of Gaussian beam of He-Ne laser pulse. In the first step, 12 fabric samples from 3 different types were irradiated by the Rhodotron accelerator TT200.

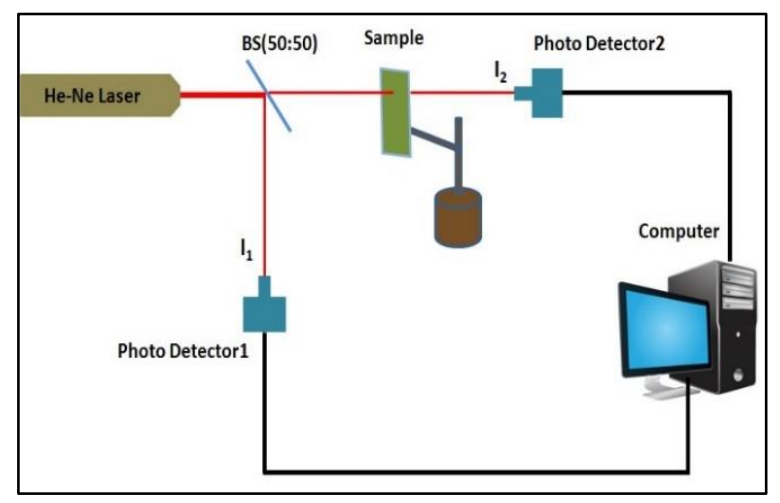

Fig. 3. Laboratory arrangement to examine changes in the absorption coefficient of aromatic fabrics

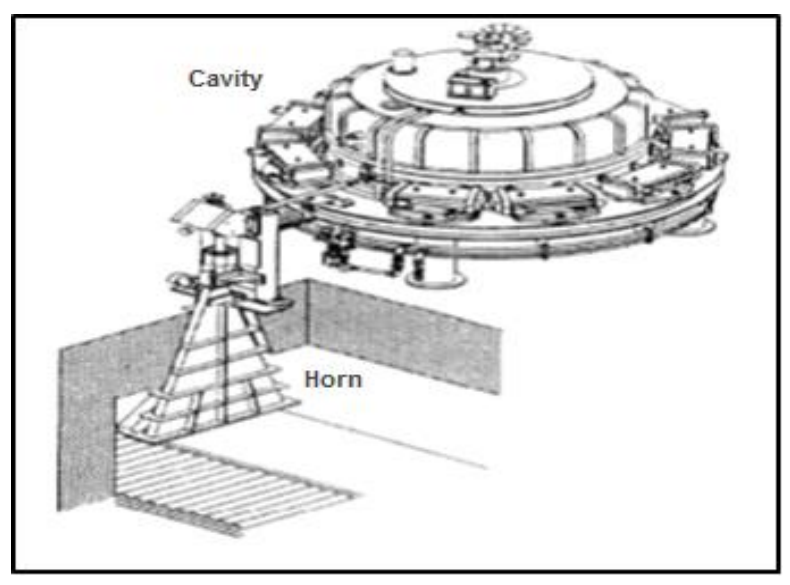

Fig. 4. Overall view of Rhodotron accelerator TT200, its transport system and scanning horn. 
Rhodotron accelerator TT200 is an industrial electron accelerator with an output of $10 \mathrm{MeV}$ beam lines and a maximum power of $100 \mathrm{~kW}$, made by IBA, Belgium [39.40].

Six containers containing three pieces of three Samples of $S_{1}, S_{2}$ and $S_{3}$ fabrics were irradiated under the Horn of Rhodotron TT200 accelerator (Fig. 4) with an energy of $10 \mathrm{MeV}$ and dosages of $5 \mathrm{kGy}, 10 \mathrm{kGy}$ and $15 \mathrm{kGy}$, three of which, in addition to the fabric samples, contained $20 \mathrm{cc}$ of the $96 \%$ ethanol. After the irradiation with a dose of $5 \mathrm{kGy}$, a sample of each fabric was removed from the containers, then again; the containers were irradiated similar to the previous process with the same parameters of the Rhodotron accelerator TT200, to reach a dose of $10 \mathrm{kGy}$. After the $10 \mathrm{kGy}$ irradiation, the second samples were removed from the containers. Similarly, the third samples were removed from the containers, after irradiation and reaching a dose of $15 \mathrm{kGy}$. Each specimen was immersed and cleaned in $200 \mathrm{cc}$ of distilled water for one minute. After the samples were cleaned and dried off, each one, with the dimensions of $20 \times 25 \mathrm{~mm}^{2}$, was installed on microscope slides. The absorption coefficient of each sample was measured at three points on their surfaces using He-Ne laser, and their mean values were recorded in Figs 5-10.

The description of irradiation procedure, conditions, dosimetry, etc. is given in Tables 1 and 2. Dosimetery was performed using CTA film (FTR-125, Fuji Film Co.).
In Table 1, the percentage of compounds is shown for the fabric samples. The following table describes the irradiation conditions of the samples.

Table 2 describes how to name the samples in empirical graphs. For example, G-5 kGy-S is a $S_{3}$ sample in the presence of alcohol and an electron beam irradiation dose of $5 \mathrm{kGy}$.

All samples of fabric were irradiated in a vessel containing $96 \%$ ethanol, finally washed with water and then immersed in a dipped antibacterial coating and perfume.

\section{Table 1}

Sample of the fabrics used as a substrate in the antibacterial coating and perfume release experiment

\begin{tabular}{llll}
\hline Compounds/samples & polyester & flannel & cotton \\
\hline $\mathrm{S}_{1}$ & $65 \%$ & $0 \%$ & $35 \%$ \\
$\mathrm{~S}_{2}$ & $0 \%$ & $100 \%$ & $0 \%$ \\
$\mathrm{~S}_{3}$ & $0 \%$ & $50 \%$ & $50 \%$ \\
\hline
\end{tabular}

\section{Table 2}

Irradiation samples labeled by e-beam in the presence/absence of alcohol

\begin{tabular}{|c|c|c|c|c|}
\hline $\begin{array}{l}\text { Absorbed } \\
\text { dose }\end{array}$ & $0 \mathrm{kGy}$ & 5 kGy & 10 kGy & 15 kGy \\
\hline $\begin{array}{l}\text { In the } \\
\text { absence of } \\
\text { alcohol }\end{array}$ & $\begin{array}{ll}0 & \text { kGy- } \\
\mathrm{S}_{\mathrm{i}} & \end{array}$ & $\begin{array}{ll}5 & \text { kGy- } \\
S_{i} & \end{array}$ & $\begin{array}{l}10 \text { kGy- } \\
S_{i}\end{array}$ & $\begin{array}{l}15 \text { kGy- } \\
S_{i}\end{array}$ \\
\hline $\begin{array}{l}\text { In the } \\
\text { presence of } \\
\text { alcohol }\end{array}$ & $\begin{array}{l}\text { G- } \quad 0 \\
k G y-S_{i}\end{array}$ & $\begin{array}{l}\text { G- } \quad 5 \\
\text { kGy- } S_{i}\end{array}$ & $\begin{array}{l}\text { G- } \quad 10 \\
k G y-S_{i}\end{array}$ & $\begin{array}{l}\text { G- } \quad 15 \\
\text { kGy- } S_{i}\end{array}$ \\
\hline
\end{tabular}

\section{RESULTS AND DISCUSSION}

For the first step of the experiment, Figures 5-7 show the rate of change in the absorption coefficient of antibacterial coating and perfumeimpregnated fabric over time, signaling the rate of the fragment-particles release in time. 


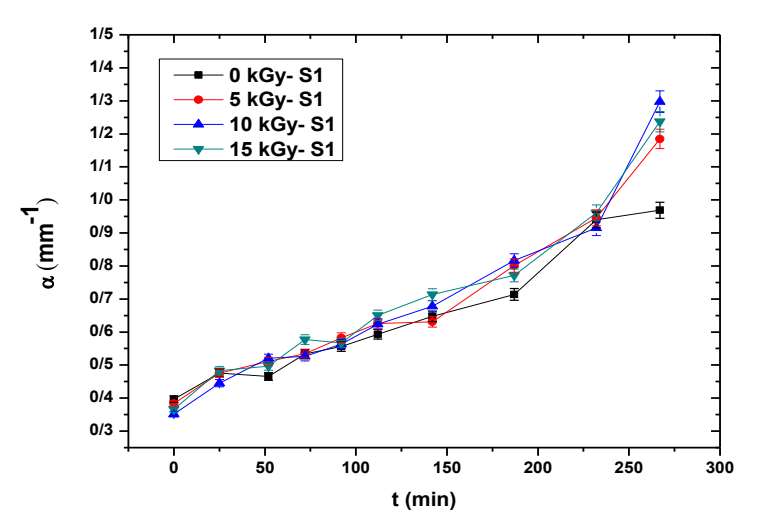

Fig. 5. Changes in the absorption coefficient of antibacterial coating and perfume-impregnated $S_{1}$ irradiated by electron beam without alcohol versus time

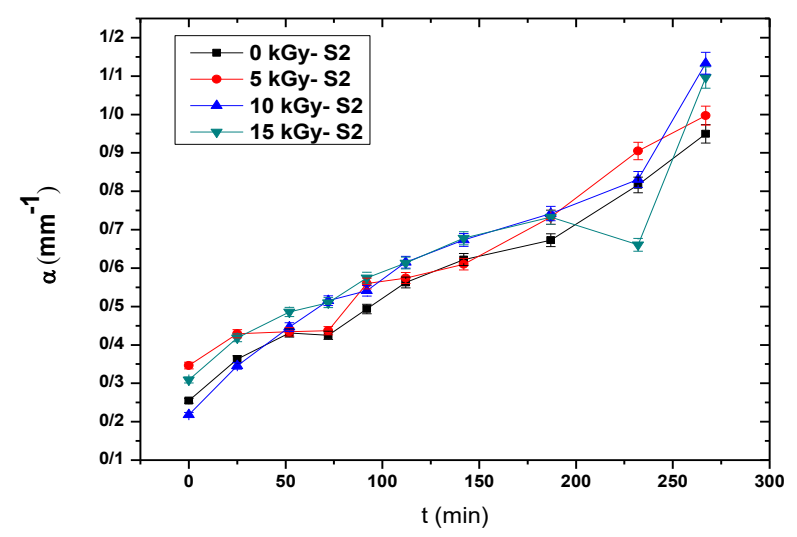

Fig. 6. Changes in the absorption coefficient of antibacterial coating and perfume-impregnated $\mathrm{S}_{2}$ irradiated by electron beam without alcohol versus time

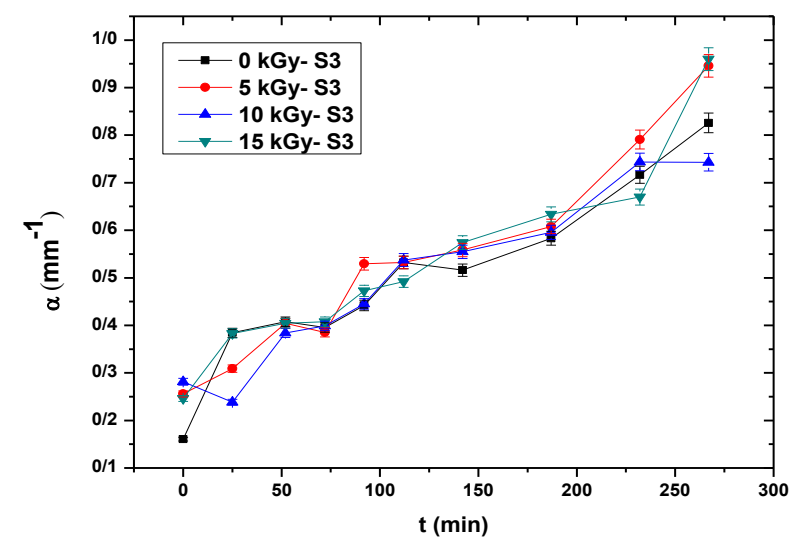

Fig. 7. Changes in the absorption coefficient of antibacterial coating and perfume-impregnated $\mathrm{S}_{3}$ irradiated by electron beam without alcohol versus time

As seen in Figures 5-7, exposing all three types of fabrics to the electron radiation does not have any significant effect on the antibacterial coating and perfume's longevity, and the release rate of antibacterial coating and perfume does not differ.

The absorption coefficients of the dry samples of $\mathrm{S}_{1}, \mathrm{~S}_{2}$ and $\mathrm{S}_{3}$ fabrics are $1.55,1.47$, and $1.38 \mathrm{~mm}^{-1}$, respectively.

For the second step of the experiment, as shown in Figures 8-10, the presence of alcohol during the time of irradiation along with the antibacterial coating and perfume-impregnated fabric significantly increases the antibacterial coating and perfume's longevity for all $\mathrm{S}_{\mathrm{i}}=1,2,3$. The electron beam radiation dose on the $S_{\mathrm{i}}=1,2$ fabrics is not very effective, but it turns out to be effective on the $\mathrm{S}_{3}$ so that for the doses higher than $5 \mathrm{kGy}$ it is seen that the electron beam irradiation has a positive effect on the antibacterial coating and perfume's longevity. However, the release of antibacterial coating and perfume for doses of $10,15 \mathrm{kGy}$ is nearly the same that is probably due to the saturation of electron radiation for all particles of the fabric. In Fig. 8, the absorption coefficient of the samples for the radiation dose of $5 \mathrm{kGy}$ over time is always higher than those shown in the two other diagrams, signaling that the physical or chemical process for this dose has not reached the saturation point. It means that for doses greater than $5 \mathrm{kGy}$, the amount of antibacterial coating and perfume leaving the fabric will not change and the duration that the antibacterial coating and perfume are emitted will not become longer. And therefore, any reaction and process that resulted in a prolonged spread of antibacterial coating and perfume from the fabric have reached the saturation point. 


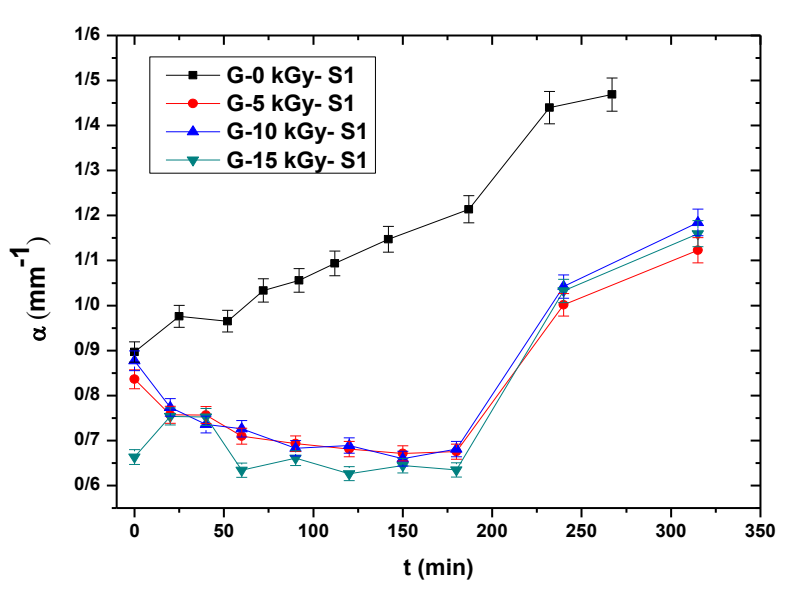

Fig. 8. Changes in the absorption coefficient of antibacterial coating and perfume-impregnated $S_{1}$ irradiated by electron beam in the presence of alcohol versus time

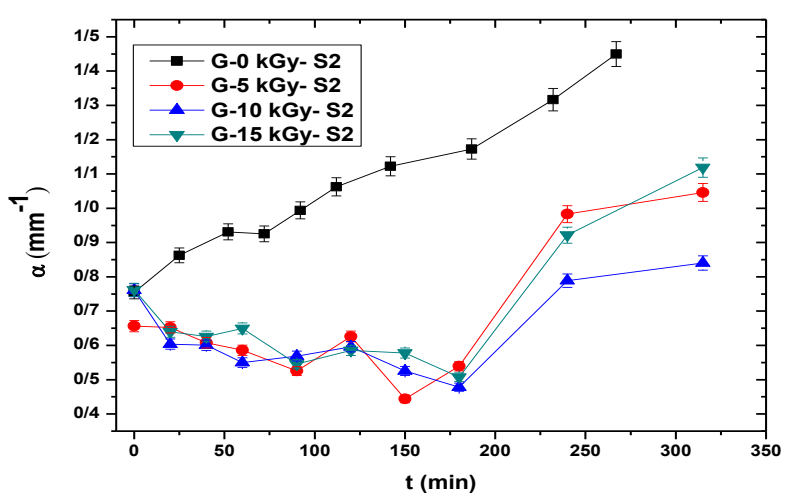

Fig. 9. Changes in the absorption coefficient of antibacterial coating and perfume-impregnated $\mathrm{S}_{2}$ irradiated by electron beam in the presence of alcohol versus time

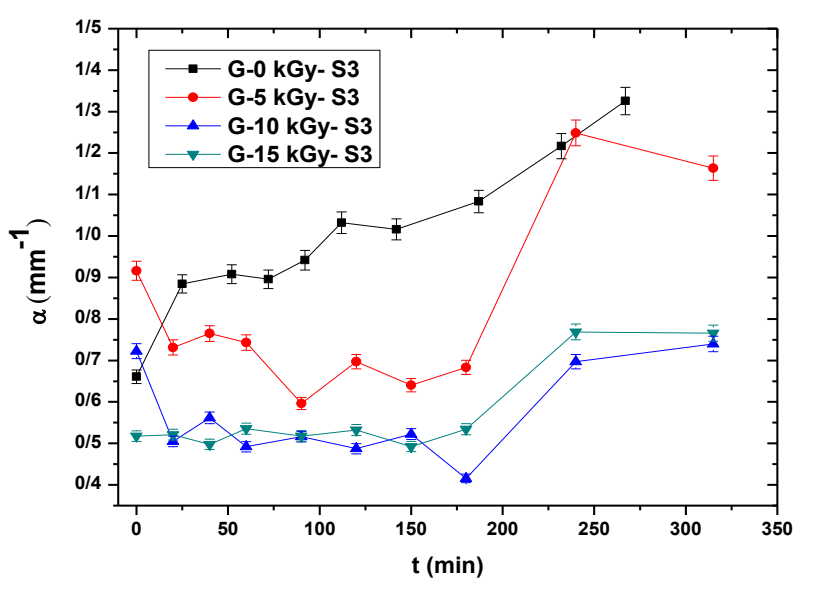

Fig. 10. Changes in the absorption coefficient of antibacterial coating and perfume-impregnated $\mathrm{S}_{3}$ irradiated by electron beam in the presence of alcohol versus time
As seen in the above Figures, the starting values (at $\mathrm{t}=0$ ) of absorption of samples irradiated in the presence of alcohol are higher than those irradiated in the absence of alcohol due to a decrease in the relative refractive index resulting from the presence of alcohol. However, both samples in the presence/absence of alcohol approach the same absorption coefficient over time. In other words, those irradiated in the presence of alcohol, and their amount of emitted antibacterial coating and perfume has increased, due to different reasons, no longer have their primary properties after the antibacterial coating and perfume are released completely. It means that if the antibacterial coating and perfume are sprayed on them again, the amount of their emitted antibacterial coating and perfume is like the specimens that have not been irradiated.

The reduction of absorbance up to 170 minutes in diagrams of Figs. 8-10 is not due to the increase in the amount of antibacterial coating and perfume, but it can be related to the difference in the swelling of the fibers of the irradiated fabric. It seems the fabrics that are irradiated in the presence of alcohol, due to the extraction of impurities and the penetration of the alcoholic $\mathrm{OH}$-functional group within the fabric tissues, have smaller holes in their surface, and this acts as a barrier against the penetration of the fragrance molecules inside them. Therefore the swelling is reduced and the duration of the penetration of the fragrance molecules increases.

As shown in the diagrams of Figs 8-10, the absorption coefficient of the irradiated samples in the presence of alcohol is approximately twice that of the irradiated samples without the presence of alcohol at time $t=0$. It means that at the time of the 
initial spraying of the antibacterial coating and perfume on the fabric samples, the fragrance molecules have not penetrated the fibers of the fabric in the irradiated samples in the presence of alcohol as done in the irradiated ones without the presence of alcohol.

Another factor that can cause longer antibacterial coating and perfume persistence and can reduce the absorbance up to 170 minutes on the samples irradiated in the presence of alcohol, is the suitable bonding of the functional groups of fabric surfaces with the different fragrance notes. Antibacterial coating and perfumes are made in such a way that their release over time, like a musical metaphor, has three notes: top notes, middle notes, and base notes [41,42]. Top notes include small and lightweight molecules that quickly evaporate and can be sensed immediately after the antibacterial coating and perfume are sprayed. Middle notes include molecules that evaporate after the release of top notes of the antibacterial coating and perfume and form the main body of the fragrance. Base notes begin to emerge after the middle notes are faded, and these compounds of this class of scents are typically rich and deep.

when the antibacterial coating and perfume are applied to the fibers of the textile. the fabric is wet with the antibacterial coating and perfume, the fabric looks more transparent and its absorption coefficient decreases. The slow and steady chemical bond of different notes of antibacterial coating and perfume with the functional groups of fabric surfaces can make the fabric clearer and reduce its absorption coefficient. Due to the separation of various notes of antibacterial coating and perfume over time, the gradient of the changes in the refractive index of air-wet fabric-air is reduced up to 170 minutes. Naturally, the absorption coefficient also decreases and after 170 minutes, when the fabric is dried, both the gradient and absorption coefficient will increase. In samples that have been irradiated without the presence of alcohol, the notes of the antibacterial coating and perfume will go through the drying process from the very beginning and their absorption coefficient will increase over time, due to the lack of proper bonding between the fragrance molecules and the surface of the fabric.

In fact, one of the most effective ways to increase the longevity of antibacterial coating and perfumes, without manipulating their chemical structure or their solvents, is to use suitable substrates. The preparation of appropriate substrates with plenty of pores and the formation of functional groups for the placement of fragrance molecules is the implication of irradiation. Because of the use of high-energy electron particles or secondary radiation in the irradiation method, pollutants and contaminants are removed from the initial bed, and functional groups suitable for the acceptance of new molecules are produced on the surface $[28,30]$.

To verify the accuracy of the experiments using the Scanning microscope electron (SEM, EGA । TESCAN, Czech), the following photographs were taken from two $\mathrm{S} 3$ cloth samples. One sample was soaked in ethanol $96 \%$ without any kind of irradiation for 1 minute, then washed twice with distilled water and then dried at $35^{\circ} \mathrm{C}$ temperature. The other sample was first irradiated at a dose of 5 kGy in the vicinity of ethanol $96 \%$ and then soaked in it for 1 minute, next washed twice with distilled water and finally dried at $35^{\circ} \mathrm{C}$ temperature. 


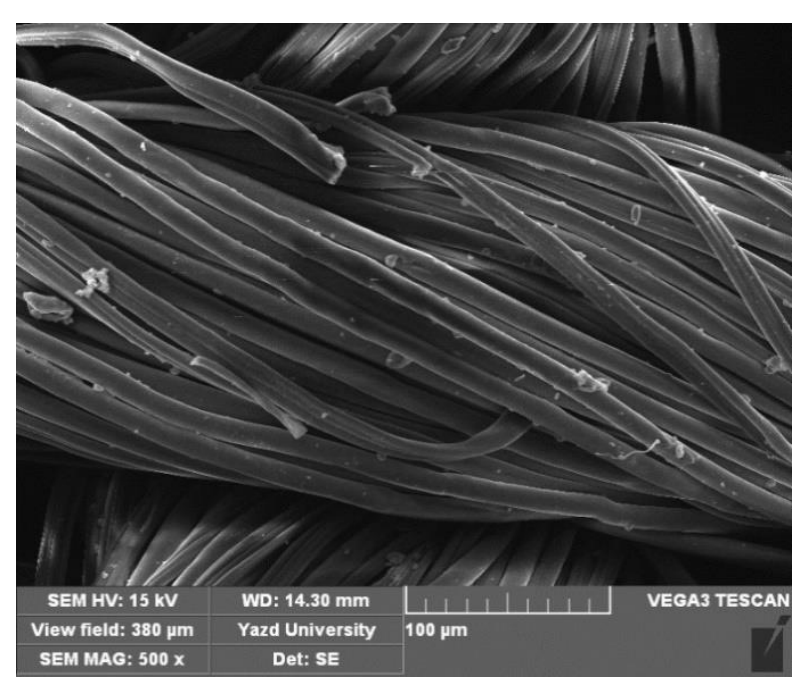

Fig. 11-a. The photo taken from the S3 sample washed without electron beam irradiation.

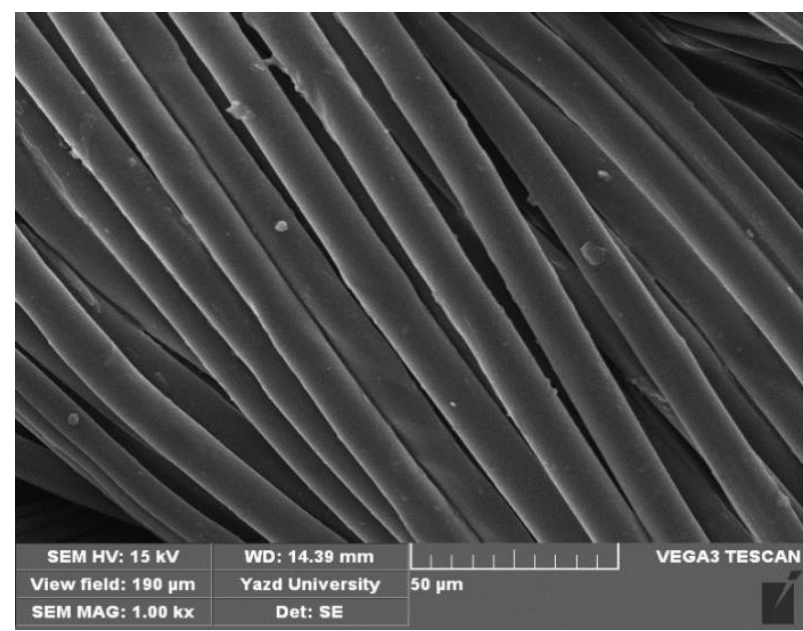

Fig. 11-b. The photo taken from the S3 sample washed with electron beam irradiation at dose of $5 \mathrm{kGy}$.

Figure 11-a shows the S3 sample on a scale of $100 \mu \mathrm{m}$, that even after being washed in ethanol 96\%, still has impurities and contamination whereas the same sample due to irradiation in the presence of alcohol, even at a smaller scale, of 50 $\mu \mathrm{m}$ is less contaminated, as shown in Fig 11-b. Using electron irradiation with a small dose in the presence of alcohol showed that this method is useful for prolonging the antibacterial coating and perfume release duration and cleansing the fabric. As shown in Fig 11-a even after washing the fabric, the amount of contamination is much higher than the sample irradiated in the presence of alcohol, shown in Fig 11-b. Therefore, it is very effective to wash the fabric with the use of electron irradiation in the presence of alcohol.

Fragrance molecules can react with these functional groups in these pores and last longer. In comparison to the use of washers, this method is faster, less costly, and usable for thicker samples. In other words, because of the high penetration depth of electrons and x-rays in polymer particles, the preparation of substrates to absorb more fragrance molecules is faster and more efficient compared to the chemical washing methods.

The sensitivity of the sample $S_{3}$ is due to the presence of cotton. Natural cotton particles have a special ability to absorb and remove impurities and moisture [44]. The impurities refer to the particles of previously sprayed antibacterial coating and perfume and any other structure on the surface of the fabric or between its fibers that prevent the penetration of fragrance molecules. Therefore, the presence of about $50 \%$ cotton in the sample $\mathrm{S}_{3}$ necessitates a greater dose (greater than $5 \mathrm{kGy}$ ) to clean this type of sample and form functional groups.

The chemical structure of the cotton fiber is cellulose in Figure 12.

When this structure is in the presence of alcohol, the alcohol infiltrates as the solvent between the fiber layers and, in accordance with the proposed mechanism bellow, the -OH groups, which can be deprotonated readily, are removed by electron beam irradiation from the cellulose structure and produce stable radicals. 


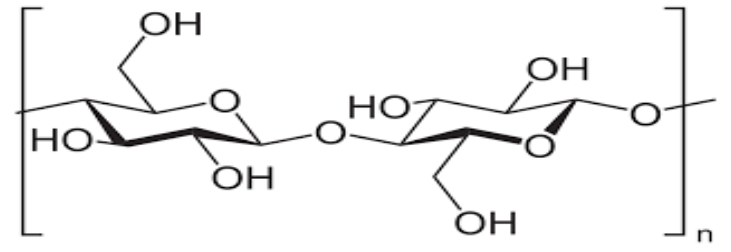

Fig 12. Chemical structure of cotton fiber

As shown in Figure 13, relatively stable free radicals type $2 \circ$ are formed during e-beam irradiation that, when exposed to a antibacterial coating and perfume with an ester structure, form a relatively strong covalent bond and reduce the release rate of the antibacterial coating and perfume. Electron irradiation with a dose of $5 \mathrm{kGy}$, can disintegrate a small amount of S3 fibers.

Figure 14-a shows the S3 sample on a scale of $500 \mu \mathrm{m}$ before being irradiated by the electron beam. But as shown in Fig. 14-b, the $S_{3}$ sample is degraded after electron irradiation at a dose of 5 kGy but not much, such that this degradation reduces only $8 \%$ of the tensile strength of the $S_{3}$ fabric.

There is also cotton in combination with polyester in the sample $S_{1}$. In other words, the texture of the sample is identical, and the fiber is a combination of cotton and polyester. In the sample $\mathrm{S}_{3}$, on the other hand, the cotton is not combined with flannel and is used in the form of isolated fibers perpendicular to the flannel. Accordingly, the effect of cotton on increasing the antibacterial coating and perfume durability is evident in the sample $S_{3}$.

Therefore, using the He-Ne laser based on the arrangement of Fig. 3, one can measure the release of the antibacterial coating and perfume from the fabric rather precisely and use it for further research. Although the laser is not able to distinguish and identify the rate of different particles released from the fabric, the overall understanding of fragrance molecules release with time is well obtained. In addition, the presence of alcohol particles adjacent to the fabric during irradiation causes the trapping of fine particles of the dirty material and the cleaning of its pores [45]. The fragrance molecules are replaced in these pores after immersion and hence released over time more slowly compared to the case without the alcohol.
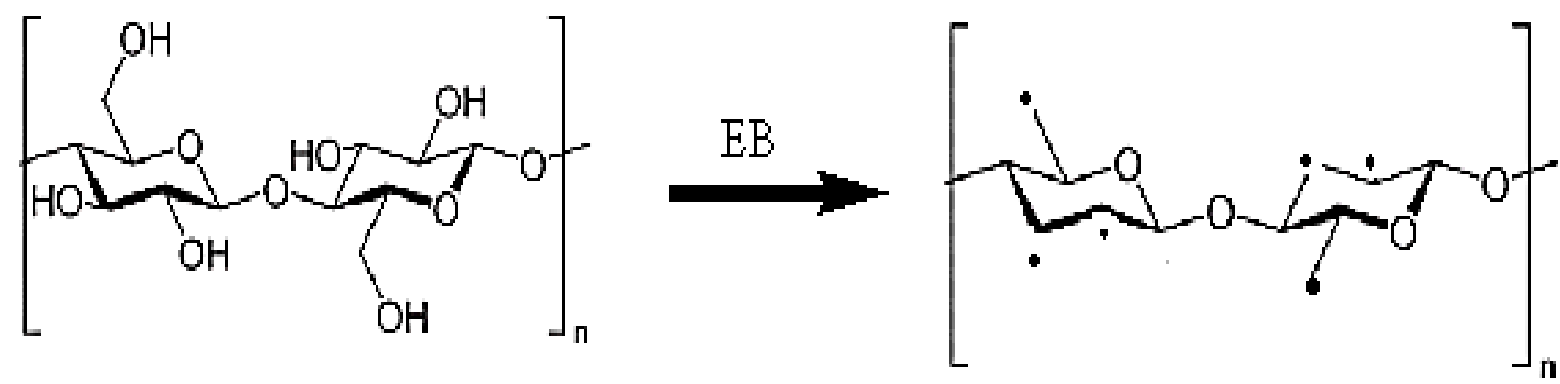

Fig. 13. The electron beam irradiation mechanism on cotton fibers 

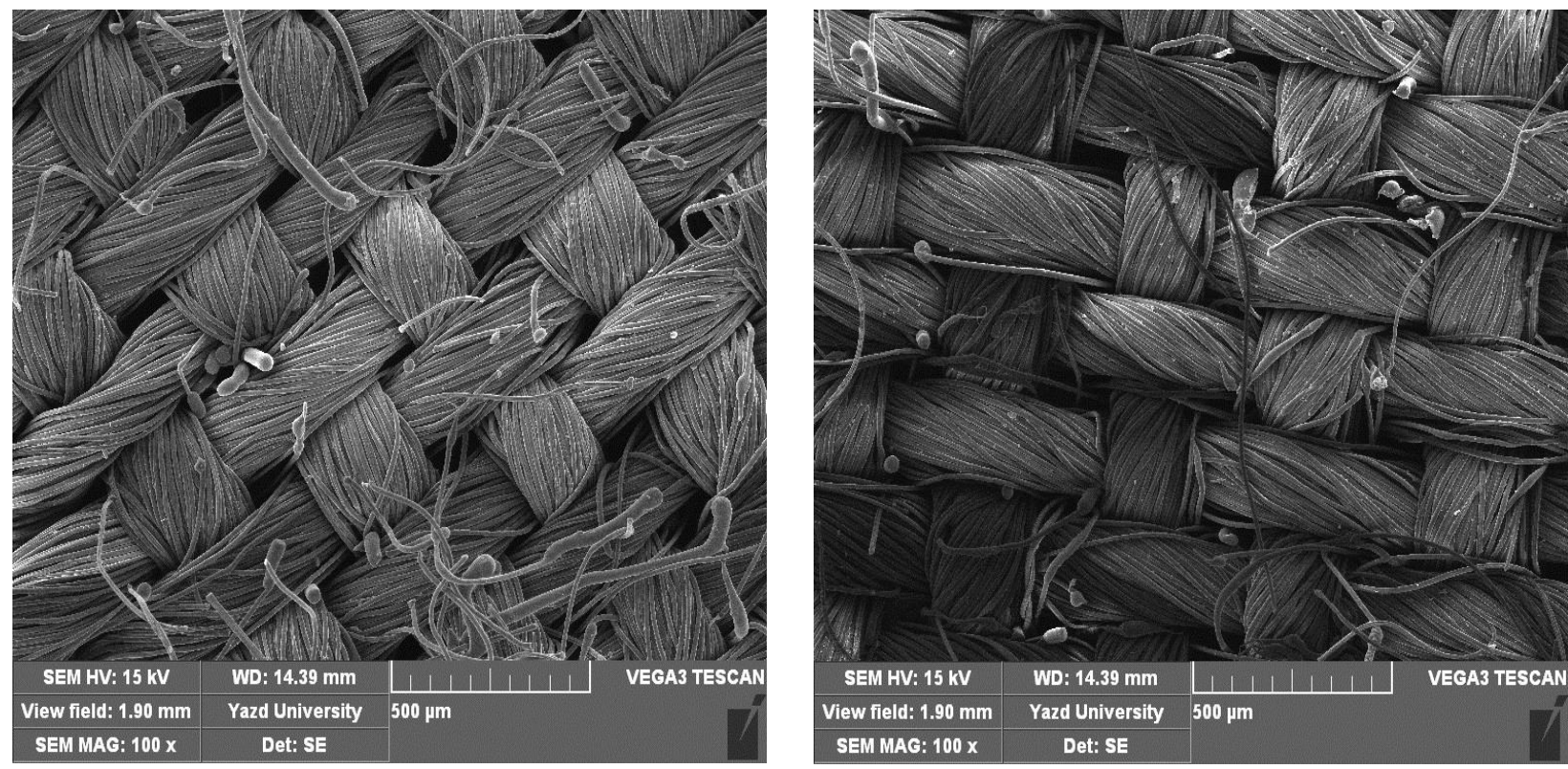

Fig. 14-a. $S_{3}$ sample fiber before electron beam irradiation Fig 14-b. $S_{3}$ sample fiber after electron beam irradiation

\section{CONCLUSION}

In this paper, we presented a new method to increase the antibacterial coating and perfume's longevity as well as to measure them. Instead of using syntheses and chemical compounds, the electron beam irradiation on the fabric which is mainly antibacterial coating and perfume substrate was performed, and for measuring the antibacterial coating and perfume longevity, the opacity property of the fabric in the presence of secondary particles was used. . The focus of this work was the modification of fabrics by the electron beam to provide them with the capability of adsorbing and slowly releasing the fragrance, as a simple method compared to the other ones. Although in this work, the laser cannot distinguish and identify the rate of different molecules released from the fabric, but the overall understanding of fragrance molecules release with time is well gained.

The obtained results showed that electronbeam irradiation of the fabric without the presence of alcohol had no effect on the antibacterial coating and perfume longevity, but this property was improved in the presence of alcohol. Samples that are irradiated in the presence of alcohol lose their impurities, and their surface becomes prepared to absorb antibacterial coating and perfume molecules due to the formation of free radicals type $2^{\circ}$ and then reaction with ester groups on the antibacterial coating and perfume, which form a covalent bond and reduce the release rate. Increasing the radiant dose adjacent to alcohol was only effective for the type $S_{3}$ of fabrics and did not affect the other ones. 


\section{REFERENCES}

1. Kuhnt, T., et al., Functionalized cellulose nanocrystals as nanocarriers for sustained fragrance release. Polymer Chemistry, 2015. 6(36): p. 6553-6562.

2. Frérot, E., K. Herbal, and A. Herrmann, Controlled Stepwise Release of Fragrance Alcohols from Dendrimer-Based 2Carbamoylbenzoates by Neighbouring Group Participation. European Journal of Organic Chemistry, 2003. 2003(6): p. 967-971.

3. Lage Robles, J. and C.G. Bochet, Photochemical release of aldehydes from $\alpha$-acetoxy nitroveratryl ethers. Organic letters, 2005. 7(16): p. 35453547.

4. Soottitantawat, A., et al., Microencapsulation of 1-menthol by spray drying and its release characteristics. Innovative Food Science \& Emerging Technologies, 2005. 6(2): p. 163-170.

5. Levrand, B., et al., Controlled release of volatile aldehydes and ketones by reversible hydrazone formation - "classical" profragrances are getting dynamic. Chemical Communications, 2006(28): p. 2965-2967.

6. Feczkó, T., V. Kokol, and B. Voncina, Preparation and characterization of ethylcellulose-based microcapsules for sustaining release of a model fragrance. Macromolecular research, 2010. 18(7): p. 636640.

7. Sansukcharearnpon, A., et al., High loading fragrance encapsulation based on a polymerblend: preparation and release behavior. International journal of pharmaceutics, 2010. 391(1): p. 267-273.

8. Tzhayik, O., A. Cavaco-Paulo, and A. Gedanken, Fragrance release profile from sonochemically prepared protein microsphere containers. Ultrasonics sonochemistry, 2012. 19(4): p. 858863.

9. Ciobanu, A., et al., Cyclodextrin-intercalated layered double hydroxides for fragrance release. Journal of Inclusion Phenomena and Macrocyclic Chemistry, 2013. 75(3-4): p. 333-339.

10. Vaughn, J., et al., Encapsulated recyclable porous materials: an effective moisture-triggered fragrance release system. Chemical Communications, 2013. 49(51): p. 5724-5726.

11. Ferrero Vallana, F.M., et al., Ionic liquids as modulators of fragrance release in consumer goods. New Journal of Chemistry, 2016. 40(12): p. 9958-9967.

12. Li, Y., et al., Heat-resistant sustained-release fragrance microcapsules. Journal of Applied Polymer Science, 2014. 131(7).
13. Li, Y., et al., Comparison of Release Behaviors of Fragrance/Hydroxypropyl- $\beta$-cyclodextrin Inclusion Complex and Fragrance Microcapsules. Integrated Ferroelectrics, 2014. 152(1): p. 81-89.

14. Liu, C. and K. Hayashi, Visualization of controlled fragrance release from cyclodextrin inclusion complexes by fluorescence imaging. Flavour and Fragrance Journal, 2014. 29(6): p. 356-363.

15. Kuhnt, T., et al., Controlled fragrance release from galactose-based pro-fragrances. RSC Advances, 2014. 4(92): p. 50882-50890.

16. Cao, Z., et al., Synthesis of fragrance/silica nanocapsules through a sol-gel process in miniemulsions and their application as aromatic finishing agents. Colloid and Polymer Science, 2015. 293(4): p. 1129-1139.

17. Hosseinkhani, B., et al., Novel biocompatible nanocapsules for slow release of fragrances on the human skin. New biotechnology, 2015. 32(1): p. 40-46.

18. Uhde, E. and N. Schulz, Impact of room fragrance products on indoor air quality. Atmospheric Environment, 2015. 106: p. 492-502.

19. Sánchez-Navarro, M.M., et al., Scent properties by natural fragrance microencapsulation for footwear applications. Polymer International, 2015. 64(10): p. 1458-1464.

20. Tylkowski, B., et al. Photo-Triggered Microcapsules. in Macromolecular Symposia. 2016. Wiley Online Library.

21. Lee, H., et al., Encapsulation and enhanced retention of fragrance in polymer microcapsules. ACS applied materials \& interfaces, 2016. 8(6): p. 4007-4013.

22. Bashari, A., N. Hemmatinejad, and A. Pourjavadi, Smart and Fragrant Garment via Surface Modification of Cotton Fabric With Cinnamon Oil/Stimuli Responsive PNIPAAm/Chitosan Nano Hydrogels. IEEE transactions on nanobioscience, 2017. 16(6): p. 455-462.

23. Rubio, O.D., et al., Anti-odour and antibacterial fabric in textile goods. 2018, Google Patents.

24. Ega, S.K., S.K. Ghosh, and B. Mallik, Nanoparticulate capsules and emulsions thereof including fragrance by emulsion polymerization. 2017, Google Patents.

25. Chen, J., Y.-C. Nho, and J.-S. Park, Grafting polymerization of acrylic acid onto preirradiated polypropylene fabric. Radiation Physics and Chemistry, 1998. 52(1-6): p. 201-206.

26. Kumar, V., et al., Radiation-induced grafting of vinylbenzyltrimethylammonium chloride (VBT) onto cotton fabric and study of its anti-bacterial activities. Radiation Physics and Chemistry, 2005. 73(3): p. 175-182. 
27. Yang, J.M., et al., Wettability and antibacterial assessment of chitosan containing radiationinduced graft nonwoven fabric of polypropyleneg-acrylic acid. Journal of Applied Polymer Science, 2003. 90(5): p. 1331-1336.

28. Kavakl1, P.A., et al., Radiation-induced graft polymerization of glycidyl methacrylate onto PE/PP nonwoven fabric and its modification toward enhanced amidoximation. Journal of applied polymer science, 2007. 105(3): p. 15511558.

29. Blouin, F.A. and J.C. Arthur Jr, The effects of gamma radiation on cotton: Part I: some of the properties of purified cotton irradiated in oxygen and nitrogen atmospheres. Textile Research Journal, 1958. 28(3): p. 198-204.

30. Demint, R.J. and J.C. Arthur Jr, the effects of gamma radiation on cotton: Part III: base exchange properties of irradiated cotton. Textile Research Journal, 1959. 29(3): p. 276-278.

31. Blouin, F.A. and J.G. Arthur Jr, The Effects of Gamma Radiation on Cotton: Part V: PostIrradiation Reactions. Textile Research Journal, 1963. 33(9): p. 727-738.

32. Reinhardt, R.M. and J.A. Harris, Ultraviolet Radiation in Treatments for Imparting Functional Properties to Cotton Textiles 1. Textile Research Journal, 1980. 50(3): p. 139-147.

33. Porter, B.R., et al., Effects of gamma, highenergy electron, and thermal neutron radiations on the fibrillar structure of cotton fibers. Textile Research Journal, 1960. 30(7): p. 510-520.

34. Teszler, O. and H.A. Rutherford, The Effect of Nuclear Radiation on Fibrous Materials: Part I: Dacron Polyester Fiber'1. Textile Research Journal, 1956. 26(10): p. 796-801.

35. Södergård, A., Perspectives on modification of aliphatic polyesters by radiation processing. Journal of bioactive and compatible polymers, 2004. 19(6): p. 511-525.
36. Hongwang, Q. and G. Huang, Mechanical behaviors of glass/polyester composites after UV radiation. Journal of Composite Materials, 2011. 45(19): p. 1939-1943.

37. Lekner, J. and M.C. Dorf, Why some things are darker when wet. Applied Optics, 1988. 27(7): p. 1278-1280.

38. Hopkins, D.N., M. Maqbool, and M.S. Islam, Linear attenuation coefficient and buildup factor of MCP-96 alloy for dose accuracy, beam collimation, and radiation protection. Radiological physics and technology, 2012. 5(2): p. 229-236.

39. Jongen, Y., et al. First Beam Test Results of the $10 \mathrm{MeV}, 100 \mathrm{~kW}$ Rhodotron. in Proceedings of EPAC. 1994.

40. Defrise, D., et al., Technical status of the first industrial unit of the $10 \mathrm{MeV}, 100 \mathrm{~kW}$ Rhodotron. Radiation Physics and Chemistry, 1995. 46(4-6): p. 473-476.

41. Butler, H., perfumery, in Poucher's perfumes, cosmetics and soaps. 1993, Springer Science \& Business Media. p. 55.

42. wikipedia. https://en.wikipedia.org/wiki/Perfume\#cite_note -25 .

43. Crivello, J.V., UV and electron beam-induced cationic polymerization. Nuclear Instruments and Methods in Physics Research Section B: Beam Interactions with Materials and Atoms, 1999. 151(1-4): p. 8-21.

44. Hoffman, G., et al., Water Relations and Growth of Cotton as Influenced by Salinity and Relative Humidity 1. Agronomy Journal, 1971. 63(6): p. 822-826.

45. Ashkenani, H., et al., Preconcentration, speciation and determination of ultra-trace amounts of mercury by modified octadecyl silica membrane disk/electron beam irradiation and cold vapor atomic absorption spectrometry. Journal of hazardous materials, 2009. 161(1): p. 276-280.

How to cite this article

M. Askarbioki, M. B. Zarandi, S. Khakshournia, et al, Effect of fabric electron radiation on increasing the antibacterial coating and perfume longevity, Journal of Nuclear Science and Applications 2 (1): 30-44 (2022), DOI: 10.24200/jon.2022.1011

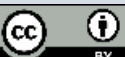

This work is licensed under the Creative Commons Attribution 4.0 International License.

To view a copy of this license, visit http://creativecommons.org/licenses/by/4.0 